\title{
The Largest Crystal Structure of a Gold Nanoparticle to date - II: $\operatorname{Au}_{246}\left(\mathrm{SC}_{6} \mathrm{H}_{4} \mathrm{Me}\right)_{80}$
}

Kristin Kirschbaum, Chenjie Zeng, Yuxiang Chen, Kelly J. Lambright, Rongchao Jin

We present here the crystal structure of the largest gold nanoparticle having 246 gold atoms and 80 surface-protecting thiolate ligands ${ }^{1}$ - the particle contains more than 100 additional Au atoms than the formerly largest structures of the $\mathrm{Au}_{133}(\mathrm{SR})_{52}$ nanoparticles we and others published in 2015. , $^{2,3}$

Nanoparticles are generally about $1-100 \mathrm{~nm}$ in size, similar to proteins. Significant efforts have been exerted to uncover the structure of these nanoparticles, because many applications require structural details at the atomic level. Crystallization as well as the crystal structure analysis of these particles are challenging, but necessary, if insight into the structural details is sought.

The here presented Au246 nanoparticle was synthesized by a two-step "size-focusing" method. Single crystals were grown by diffusion of antisolvent (acetonitrile) into a toluene solution of the pure Au246 nanoparticles. Crystallographic analysis was performed in-house using a Bruker Apex Duo (I $\mu \mathrm{S} \mathrm{CuK} \alpha, 100 \mathrm{~K}$, $0.96 \AA$ resolution) diffractometer. The structure of the gold cluster was solved and refined in $\mathrm{C} 2 / \mathrm{c}$ with $\mathrm{Z}=4$. The final refinement with 2631 variables and 1107 restraints converged at $R_{1}=6.94 \%$ for the observed data. Challenges of the refinements, strategies, peculiarities and necessity as well as disadvantages of restraints/constraints are discussed.

The individual nanoparticles have a nearly spherical shape, with a metal core diameter of $2.2 \mathrm{~nm}$ and an overall diameter (including the ligand shell) of $3.3 \mathrm{~nm}$. The complexity of the core/shell structure and the ligand arrangement parallels that of proteins.

(1) Zheng, Jin, Kirschbaum, et al. Science 2016, 356, 1580

(2) Dass, Noll et al. 2015, J. Am. Chem. Soc., 137, 4610

(3) Zeng, Jin, Kirschbaum et al. 2015, Science Advances, 1, e1500045

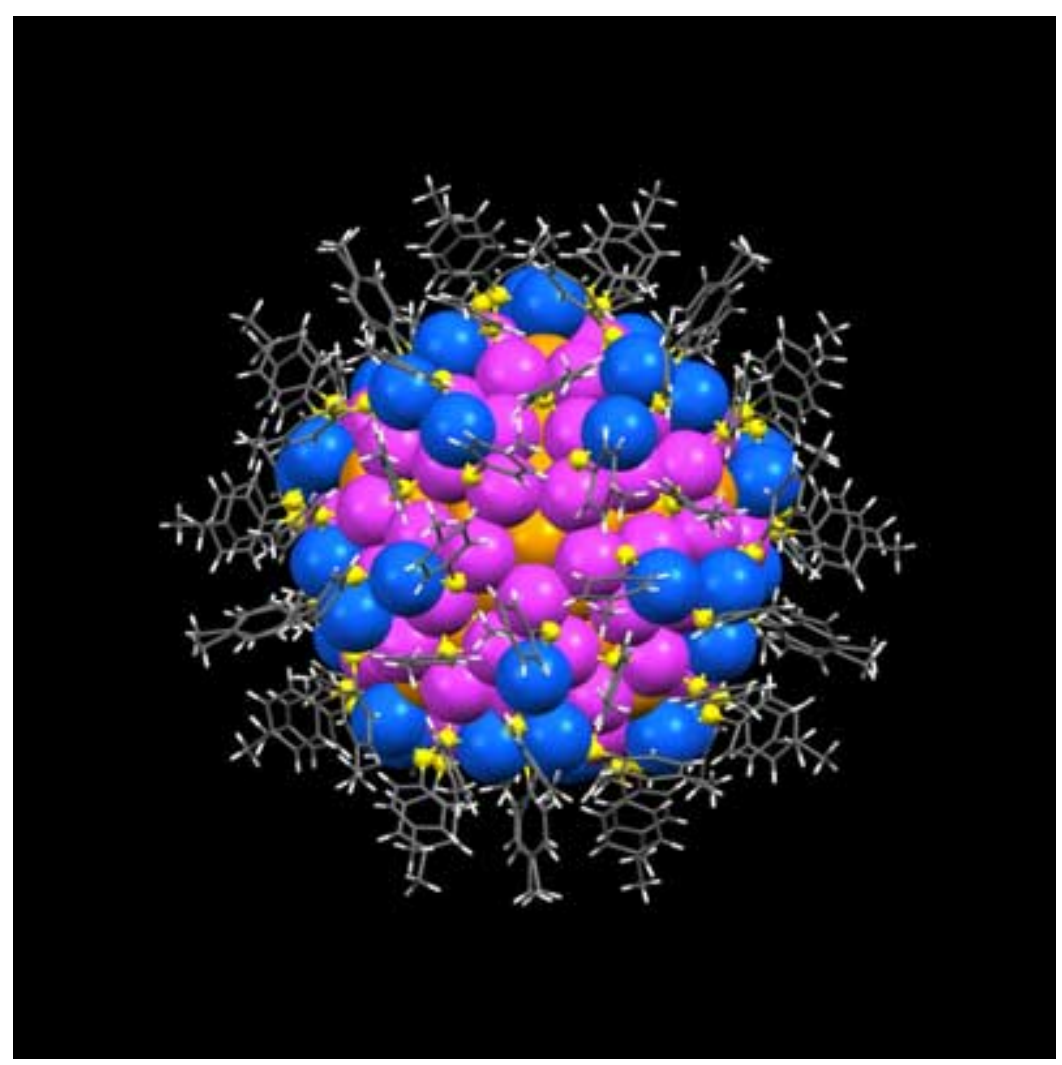

